\title{
Influência das condições térmicas do galpão de espera climatizado na mortalidade pré-abate de frangos de corte
}

\author{
[The influence of thermal conditions from an environmentally controlled preslaughter lairage on \\ mortality of broiler chickens] \\ F.M.C. Vieira ${ }^{1}$, I.J.O. Silva ${ }^{2}$, J.A.D. Barbosa Filho ${ }^{3}$, A.M.C. Vieira ${ }^{4}$ \\ ${ }^{1}$ Universidade Tecnológica Federal do Paraná - Dois Vizinhos, PR \\ ${ }^{2}$ USP-ESALQ - Piracicaba, SP \\ ${ }^{3}$ Universidade Federal do Ceará - Fortaleza, CE \\ ${ }^{4}$ UFSCar - São Carlos, SP
}

\section{RESUMO}

Avaliou-se o efeito das condições ambientais da espera pré-abate na mortalidade de frangos de corte, mantidos em galpão climatizado em um abatedouro comercial. Foram avaliados 215 caminhões de transporte de um abatedouro comercial quanto ao número de aves mortas durante as operações pré-abate e à temperatura retal dos animais em condição de espera no abatedouro. Os dados foram obtidos no galpão de espera climatizado por meio de ventiladores e nebulizadores, em diferentes períodos: manhã, tarde e noite. As variáveis térmicas, distância, tempo de espera e densidade de aves por caixa foram consideradas na análise. Destacaram-se os efeitos da temperatura, da umidade relativa e do tempo de espera na variação tanto do número de aves mortas por caminhão quanto da temperatura retal dos animais. Com relação à interação entre temperatura e umidade relativa interna do galpão de espera, houve eficiência do ambiente climatizado no abatedouro quanto à diminuição do número de aves mortas. A climatização foi mais expressiva na redução do número de aves mortas e da temperatura retal quando a temperatura do galpão de espera estava entre 21 e $24^{\circ} \mathrm{C}$, principalmente em condições de umidade relativa interna em torno de $70 \%$. Nessas condições climatizadas, o tempo de espera acima de duas horas proporcionou maior conforto às aves.

Palavras-chave: abatedouro, avicultura, mortes na chegada, operações pré-abate

\begin{abstract}
The effect of environment conditions of preslaughter lairage on broiler mortality was assessed in the present study. Daily preslaughter data from 215 broiler flocks were recorded regarding the number of dead chickens during preslaughter operations and rectal temperature of broilers submitted to a lairage environment prior to slaughtering. A holding area at a slaughterhouse with environmental control was assessed. The thermal variables, distance between farms and slaughterhouse, lairage time and density of birds per cage was considered in this analysis. The effect of dry-bulb temperature, relative humidity and lairage time on death before arrival and rectal temperature was highlighted in this assessment. Regarding the interaction between temperature and inside relative humidity, a decrease in preslaughter mortality was observed due to exposure to a controlled environment. The environment control promoted an expressive reduction in dead birds and rectal temperature when the temperature inside the holding area was between 21 and $24{ }^{\circ} \mathrm{C}$, mainly with inside relative humidity around $70 \%$. In these environment control conditions lairage above 2 hours was necessary to reduce the thermal stress of birds.
\end{abstract}

Keywords: death on arrival, poultry production, preslaughter operations, slaughterhouse

\section{INTRODUÇÃO}

A condição térmica em regiões de clima tropical tornou-se um desafio para o avanço da avicultura. Como resultado dos elevados valores médios de temperatura e umidade relativa do ar, aumentaram-se as proporções de perdas por mortalidade, principalmente durante as operações pré-abate, que correspondem à pega das aves, ao carregamento, ao transporte e à espera no abatedouro.

Recebido em 29 de julho de 2014

Aceito em 16 de setembro de 2015

E-mail: fredericovieira@utfpr.edu.br 
Durante as etapas que antecedem o abate, $40 \%$ das perdas que ocorrem são relacionadas ao estresse térmico, seja por frio ou calor (Ritz et al., 2005). Devem-se também levar em consideração os núcleos térmicos de calor dentro da carga, os quais agravam o quadro de estresse das aves em trânsito (Mitchell e Kettlewell, 1998; Barbosa Filho et al., 2009).

O efeito do transporte sobre as aves desencadeia diversos mecanismos fisiológicos como resposta ao estresse sofrido durante o trajeto, afetando as reservas energéticas e provocando hemorragias, problemas no metabolismo e mortalidade (Kranen et al., 1998; Bressan e Beraquet, 2002; Gregory, 2010). Segundo Simões et al. (2009), a ocorrência de certos problemas ligados à qualidade da carne, como, por exemplo, a carne PSE (pálida, mole e exsudativa), referem-se às viagens longas e a regiões do meio e fundo dos caminhões. Os mesmos autores explicaram que, nessas regiões, as condições térmicas de temperatura, umidade relativa e velocidade do vento são críticas para as aves, favorecendo distúrbios metabólicos que prejudicam a qualidade do produto final.

As condições térmicas são inaceitáveis, atingindo proporções de mortalidade acima de $1 \%$ em certos trechos da viagem (Kettlewell et al., 2000). Quando transportadas em conforto térmico (entre 15 e $22^{\circ} \mathrm{C}$ ), as aves não apresentaram alterações nos parâmetros fisiológicos. Todavia, as aves mantidas durante duas horas em caixas de transporte a $34^{\circ} \mathrm{C}$ evidenciaram quadro de hipertermia (Akşit et al., 2006). Dessa forma, os animais chegam fisiologicamente comprometidos ao abatedouro, e a permanência no galpão de espera assume um papel fundamental no reequilíbrio térmico das aves recém-chegadas do transporte devido ao efeito da climatização (Vieira et al., 2010; 2011a). Não basta a preocupação apenas com o espaço onde os caminhões permanecerão estacionados durante o tempo de espera; também é necessário o cuidado com o ambiente térmico que facilite as trocas entre os animais e o meio. A adequação e o controle ambiental no galpão de espera são importantes, a fim de se reduzir a carga térmica do ambiente e dos animais (Ritz et al., 2005; Silva e Vieira, 2012).

Objetivou-se, por meio desta pesquisa, avaliar o efeito das condições ambientais e do tempo de espera pré-abate na mortalidade e na temperatura retal de frangos de corte, mantidos em galpão climatizado em um abatedouro comercial.

\section{MATERIAL E MÉTODOS}

A pesquisa foi conduzida em um abatedouro comercial de frangos de corte, situado na latitude $22^{\circ} 01^{\prime} 03^{\prime} \mathrm{S}$, longitude $47^{\circ} 53^{\prime} 27^{\prime}$ ' $\mathrm{W}$ e altitude média da região de $856 \mathrm{~m}$. O clima local é caracterizado como tropical de altitude Cwa, segundo a classificação de Köppen, com temperatura do mês mais frio inferior a $18^{\circ} \mathrm{C}$ e a do mês mais quente superior a $22^{\circ} \mathrm{C}$ (Embrapa Pecuária Sudeste, 2007). O registro de dados foi realizado no galpão de espera do abatedouro, entre os meses de outubro de 2006 e março de 2007, sendo avaliados 215 caminhões de transporte estacionados sob a condição de espera, além de planilhas de dados da empresa.

O ambiente de espera no abatedouro foi caracterizado tipologicamente por um galpão de 23,70m de comprimento, 19,22m de largura, pé direito de $5 \mathrm{~m}$ e beiral de $0,60 \mathrm{~m}$, totalizando uma área livre de $455,5 \mathrm{~m}^{2}$. Possuía cobertura de telhas galvanizadas, com seis tesouras metálicas. $\mathrm{O}$ ambiente era climatizado com ventiladores axiais, cuja potência era de $0,5 \mathrm{cv}$ e vazão de $300 \mathrm{~m}^{3} / \mathrm{min}$, dispostos lateralmente nos pilares (um ventilador em cada pilar) e nas tesouras da estrutura, em quatro linhas de sete ventiladores cada uma. Também possuía oito linhas de nebulização, cada uma com 25 bicos de alta pressão, intercaladas com as linhas de ventilação, cuja distribuição era de um bico por metro linear. As laterais dessa sala possuíam telas de polipropileno (do tipo sombrite), para sombrear e amenizar os efeitos da radiação solar direta nos caminhões.

O galpão possuía capacidade para abrigar oito caminhões de transporte de frangos. Cada caminhão possuía capacidade para 486 caixas, cada uma com uma densidade média de sete aves. As caixas de transporte apresentavam as seguintes dimensões: $75 \mathrm{~cm}$ de comprimento, $60 \mathrm{~cm}$ de largura e $30 \mathrm{~cm}$ de altura. As aves utilizadas nos transportes foram da linhagem Cobb, com idades variando entre seis e sete semanas e peso médio de $2 \mathrm{~kg}$.

Os seguintes fatores foram analisados neste estudo: turnos diários de transporte (manhã, tarde 
e noite), densidade de aves por caixa, distância granja-abatedouro, tempo de espera pré-abate, bem como os fatores térmicos envolvidos (temperatura e umidade relativa). Para este trabalho, foram evidenciados os efeitos da temperatura $\left({ }^{\circ} \mathrm{C}\right)$, da umidade relativa $(\%)$ e do tempo de espera (minutos) na variação tanto do número de aves mortas por caminhão quanto da temperatura retal dos animais.

Para a caracterização térmica da condição externa ao galpão de espera, foram utilizadas médias e desvios-padrão de temperatura do ar (Temp., ${ }^{\circ} \mathrm{C}$ ), umidade relativa do ar (UR, \%) e entalpia específica (h, $\mathrm{kJ} / \mathrm{kg}$ de ar seco).

O tempo de espera consistiu no período entre a chegada do caminhão à empresa, a permanência na espera e a saída para a linha de abate. O tempo de jejum adotado em cada carregamento foi de oito horas, e todos os intervalos de tempo adotados nas operações pré-abate (pega, carregamento, transporte e espera no abatedouro) estão contidos no período de jejum.

A temperatura retal e o número de aves mortas foram, respectivamente, variáveis de resposta fisiológica e produtiva. Para a aferição da temperatura retal, foram selecionadas aleatoriamente cinco aves em uma caixa de cada caminhão estudado. Para os registros da temperatura retal, foram realizadas duas amostragens em cada caminhão, na chegada do caminhão ao galpão de espera e na saída desse ambiente rumo à linha de abate.

A diferença entre a temperatura retal das aves da saída e da chegada ao galpão de espera foi incorporada na análise estatística (diferença da temperatura retal) com o intuito de se verificar a redução ou o aumento na temperatura retal dos animais, ocasionada pela climatização no galpão de espera. $\mathrm{O}$ número de aves mortas por caminhão foi obtido por meio de dados de perdas descritas nas planilhas de controle da empresa.

Os dados foram analisados utilizando-se os modelos lineares generalizados duplos (DGLM) (Vieira, 2008). Essa classe de modelo consiste em uma extensão dos modelos lineares generalizados (MLG), a qual visa modelar, simultaneamente, a média e a dispersão dos dados. A diferença da temperatura retal foi considerada neste estudo como uma variável resposta com distribuição normal, com sua média ligada ao preditor linear utilizando a função identidade. O número de aves mortas foi tratado como uma variável resposta com distribuição de Poisson. A função logarítmica foi assumida para fazer a função de ligação entre o preditor linear do modelo e a esperança da variável resposta. Também foi adotada, para as duas variáveis respostas, uma função logarítmica na modelagem da variabilidade dos dados, garantindo valores positivos para o parâmetro de dispersão esperado $(\hat{\phi})$.

Os modelos lineares generalizados duplos para as duas variáveis respostas foram selecionados com base nos testes de Wald, com o objetivo de testar as hipóteses sobre a contribuição real dos fatores analisados e das interações no modelo estatístico (Knight, 2000). Esse teste é uma extensão do teste $t$ de Student, largamente utilizado nas análises de regressão linear. Complementar ao teste de Wald, uma análise de resíduos foi realizada, para verificar as pressuposições dos modelos, baseada na deviance residual, a independência dos valores preditos versus ajustados, o gráfico de locação-escala e a distância de Cook, comumente utilizada na avaliação da qualidade de ajuste dos MLG (McCullagh e Nelder, 1989). O ambiente de computação estatística R (R Development Core Team, 2006) foi utilizado em conjunto com a biblioteca dglm (Dunn e Smyth, 2006) para estimação dos parâmetros dos modelos e realização dos testes de hipóteses.

\section{RESULTADOS E DISCUSSÃO}

Os maiores valores de temperatura foram registrados no turno da tarde, variando entre 24 e $35^{\circ} \mathrm{C}$, seguido do turno da manhã, com média de $25,3^{\circ} \mathrm{C}$ e variação entre 19,5 e $31,1^{\circ} \mathrm{C}$. Nota-se que, apesar da variabilidade observada em relação ao turno da noite, a temperatura mantevese controlada no ambiente climatizado, permanecendo em torno de $22^{\circ} \mathrm{C}$ (Tab. 1).

Em relação à umidade relativa, as maiores variações foram observadas nos turnos da manhã e da tarde, cujos valores foram menores do que no período noturno. No ambiente de espera no abatedouro, observou-se aumento da umidade, em razão do uso de nebulização e molhamento na carga, mantendo o ambiente próximo da 
saturação de vapor d'água. Ou seja, as aves, em termos de temperatura, mantiveram-se dentro do conforto térmico, porém numa faixa de estresse quando observados os valores de umidade relativa recomendados por diversos autores (Curtis, 1983; Silva, 2000; Macari e Furlan, 2001).

Tabela 1. Valores médios e desvios-padrão das variáveis do ambiente pré-abate de frangos de corte (externo e interno) para os turnos diários da pesquisa (manhã, tarde e noite)

\begin{tabular}{lccc} 
& \multicolumn{3}{c}{ Turnos diários } \\
\cline { 2 - 4 } Variáveis & Manhã & Tarde & Noite \\
& & $29,8 \pm 5,8$ & $23,7 \pm 2,7$ \\
\hline TExt. $\left({ }^{\circ} \mathrm{C}\right)$ & $25,3 \pm 5,8$ & $55 \pm 21$ & $82 \pm 15$ \\
URExt. (\%) & $68 \pm 23$ & $73,0 \pm 5,5$ & $68,5 \pm 4,6$ \\
hExt. $(\mathrm{kJ} / \mathrm{kg}$ ar seco) & $67,7 \pm 5,5$ & $23,3 \pm 1,5$ & $23,1 \pm 2,2$ \\
TInt. $\left({ }^{\circ} \mathrm{C}\right)$ & $20,8 \pm 1,8$ & $95 \pm 8$ & $94 \pm 8$ \\
URInt. $(\%)$ & $86 \pm 16$ & $70,4 \pm 2,7$ & $69,8 \pm 4,5$ \\
hInt. $(\mathrm{kJ} / \mathrm{kg}$ ar seco) & $63,3 \pm 2,2$ & & \\
\hline
\end{tabular}

Devido à ventilação irregular entre as caixas, a diferença de temperatura entre o ambiente interno da carga e o externo pode chegar a $8,8^{\circ} \mathrm{C}$ com o veículo parado e a $6^{\circ} \mathrm{C}$ com ele em movimento (Webster et al., 1993). Com isso, mesmo dentro de faixas de temperatura que favorecem a ave na manutenção da homeostase, a umidade do ar reduz a eficácia da termólise nas aves em até $50 \%$, sobrecarregando o metabolismo e aumentando a temperatura do núcleo corporal (Balnave, 1998).

As variáveis que apresentaram interações entre si $(\mathrm{P}<0,05)$ no modelo de mortalidade foram: tempo de espera, densidade de aves por caixa, temperatura interna e externa e umidade relativa interna e externa. No modelo da diferença da temperatura retal, houve interações entre as seguintes variáveis: tempo de espera, temperatura interna e externa, densidade de aves por caixa, períodos do dia e distância. Não houve interação entre as variáveis: período da noite vs. distância (modelo da diferença da temperatura retal); umidade relativa $v s$. período da tarde; e tempo de espera $v s$. período da noite (modelo da mortalidade).

A interação entre a temperatura e a umidade contribuiu para a estimação do número de aves mortas, evidenciando que a condição térmica é fator determinante para a quantificação de perdas durante as operações pré-abate (Tab. 2).

Tabela 2. Modelo linear generalizado duplo da interação entre temperatura interna e umidade relativa interna (modelo da média e dispersão) no ambiente pré-abate de frangos de corte

\begin{tabular}{lc} 
Componentes & Modelo $^{1}$ \\
\hline Média & $\hat{y}_{1}=\exp \left(-49,71^{*}+0,76^{*} t_{2}-0,15^{*} u_{2}-9,03 \cdot 10^{-4 *} t_{2} u_{2}\right)$ \\
Dispersão & $\hat{\phi}_{1}=\exp \left(-4,82^{*}+0,09^{*} t_{1}-2,91^{\mathrm{NS}} e^{l}-4,39^{*} e^{2}-4,01^{\mathrm{NS}} t_{2}{ }^{l}-8,69^{*} t_{2}{ }^{2}+0,04^{*} u_{2}-\right.$ \\
& $\left.0,81^{*} a-0,19^{\mathrm{NS}} n\right)$
\end{tabular}

${ }^{1} \hat{y}_{1}$ : parâmetro da média esperado (mortalidade); $\hat{\phi}_{1}$ : parâmetro de dispersão esperado (mortalidade); $a$ : turno da tarde; $n$ : turno da noite; $t_{1}$ : temperatura externa; $t_{2}$ : temperatura interna; $u_{2}$ : umidade relativa interna; $e^{I}, e^{2}$ : polinômio de segunda ordem para tempo de espera; $t_{2}{ }^{1}, t_{2}{ }^{2}$ : polinômio de segunda ordem para temperatura interna.

Com relação ao modelo da dispersão, a temperatura externa apresentou contribuição positiva para a variabilidade da mortalidade. $\mathrm{O}$ aumento de $1^{\circ} \mathrm{C}$ na temperatura do ar correspondeu ao aumento de 0,09U de variabilidade da mortalidade. A explicação para esse fato refere-se à heterogeneidade térmica existente ao longo da carga no caminhão de transporte e ao ambiente de espera no abatedouro, de acordo com trabalhos anteriores (Simões et al., 2009; Silva e Vieira, 2010). No presente trabalho, foi observado que o acréscimo 
da carga térmica no ambiente promove maior variabilidade de aves mortas ao longo da carga, isto é, existem pontos de maior ocorrência de aves mortas em contraste com outras regiões da carga. Quanto ao período do dia, o transporte realizado durante a tarde reduziu em $0,81 \mathrm{U}$ de variabilidade da mortalidade. Os resultados corroboram Vieira et al. (2011b), os quais afirmaram que as condições térmicas são pouco variáveis nesse período, ou seja, a temperatura é mais elevada e, com isso, o conforto e o bemestar para as aves são críticos. A mortalidade é elevada nesse período e pouca variação dentro dessa faixa é observada nessa situação.

Houve efeito conjugado entre temperatura do galpão de espera e do tempo de permanência dos caminhões na diferença da temperatura retal das aves (Tab. 3). Esses resultados evidenciam a importância do tempo de espera em um galpão climatizado visando à sobrevivência das aves antes da chegada à linha de abate (Bianchi et al., 2005; Vieira et al., 2010).

Tabela 3. Modelo linear generalizado duplo da interação entre tempo de espera pré-abate de frangos de corte e temperatura interna (modelo da média e dispersão)

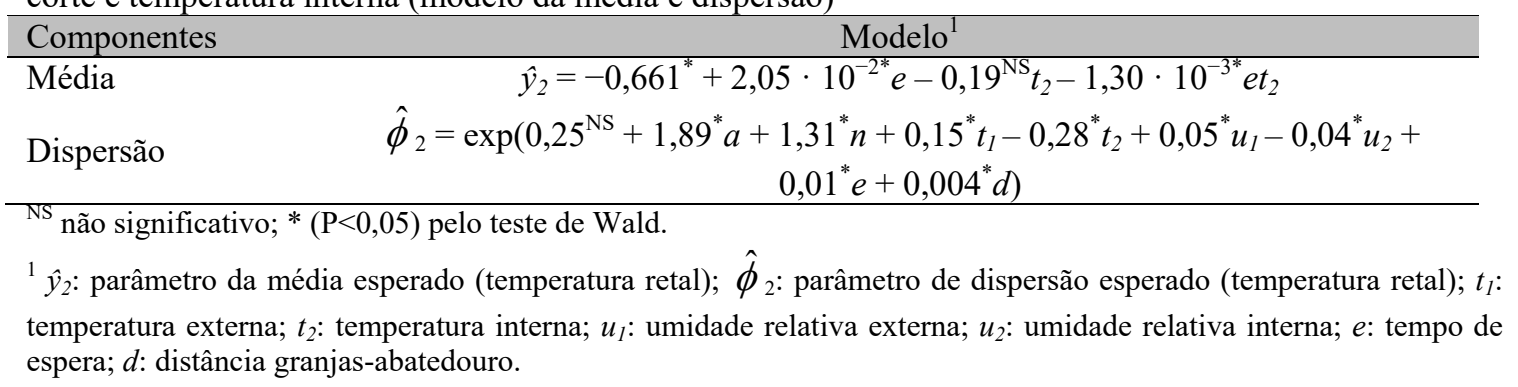

Com relação ao modelo de dispersão, destaca-se a contribuição positiva e linear do período noturno. Tal como no modelo da mortalidade, devido ao fato de a estação do ano do presente trabalho ser caracterizada por temperaturas elevadas ao longo dos meses, as condições térmicas durante $\mathrm{o}$ período noturno são constantes. Ou seja, o carregamento no período da tarde proporcionou às aves maior carga térmica do que a que se mantém no período noturno (Barbosa Filho et al., 2009). Essa condição térmica noturna semelhante ao longo dos dias resultou em redução da variabilidade da temperatura retal.

A influência conjunta das condições térmicas do galpão de espera no número de aves mortas por caminhão é evidenciada na Fig. 1.

As melhores condições foram alcançadas quando a temperatura do ambiente interno esteve entre a faixa de conforto e alerta (entre 21 e $24^{\circ} \mathrm{C}$ ), e a umidade relativa em torno de $70 \%$. Não foram verificadas condições de alerta para umidade relativa (entre 71 e 79\%), assim como condições letais para temperatura (acima de $28^{\circ} \mathrm{C}$ ). Isso é explicado pelo controle da climatização realizada no galpão, o que manteve a temperatura dentro do limite de conforto das aves. Entretanto, como a nebulização era acionada sem o controle de quantidade de água no ambiente, tanto a saturação de vapor d'água quanto valores baixos de umidade eram esperados no presente trabalho.

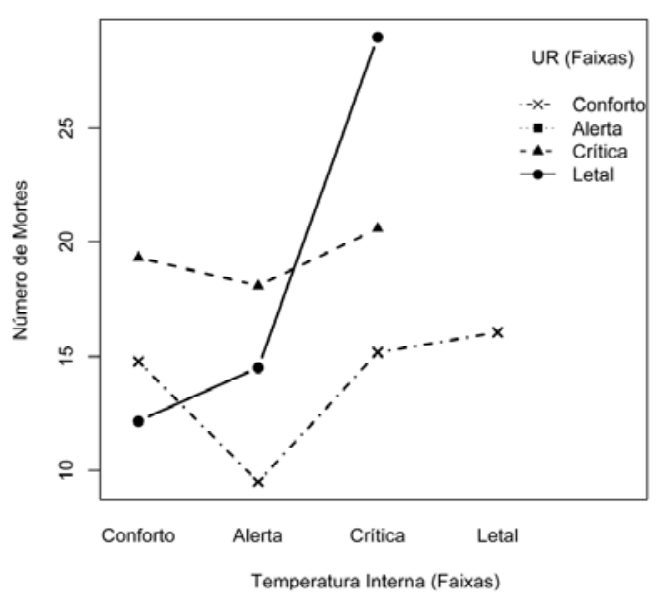

Figura 1. Valores médios da interação entre a temperatura interna e a umidade relativa interna do ambiente pré-abate de frangos de corte em relação ao número de aves mortas, sendo as faixas de temperatura: conforto: abaixo de $21^{\circ} \mathrm{C}$; alerta: $22-24^{\circ} \mathrm{C}$; crítica: $25-28^{\circ} \mathrm{C}$; letal: acima de $28^{\circ} \mathrm{C}$, e as faixas de umidade: conforto: abaixo de 70\%; alerta: $71-79 \%$; crítica: $80-$ 90\%; letal: acima de $91 \%$. 
Considera-se que o calor produzido pelas aves é alto, provocando um gradiente de temperatura dentro das caixas de transporte no caminhão (Mitchell e Kettlewell, 1994; Nijdam et al., 2004). Conforme Silva e Vieira (2012), isto é agravado pelas operações pré-abate anteriores à chegada ao abatedouro, com condições térmicas inaceitáveis sob o ponto de vista de bem-estar animal. Dessa forma, as aves necessitam de um ambiente térmico desejável durante a espera, com nebulização e ventilação suficientes para que a temperatura corporal das aves possa manter-se em um nível que não prejudique sua sobrevivência. Bayliss e Hinton (1990) registraram proporções reduzidas de mortalidade (próximas de $0,2 \%$ ) quando foi utilizado um galpão de espera climatizado durante o verão. No presente estudo, quando a temperatura e a umidade relativa estiveram na zona de conforto, a média de mortalidade foi de aproximadamente $0,35 \%$, o que confirma a eficácia do controle das condições térmicas no abatedouro para a redução de perdas.

A Fig. 2 ilustra a variação da diferença de temperatura retal em diferentes combinações de tempo de espera e temperatura do galpão de espera climatizado. Nota-se que, na faixa de conforto (abaixo de $21^{\circ} \mathrm{C}$ ), a temperatura retal pouco variou, mostrando que, nessa situação, o tempo de espera possui pouca influência. No entanto, quando a temperatura esteve entre a faixa de alerta e crítica (entre 22 e $28^{\circ} \mathrm{C}$ ), a redução foi mais efetiva quando o tempo de espera foi superior a duas horas.

Os resultados deste trabalho estão de acordo com Furlan et al. (2000), os quais encontraram redução significativa na temperatura corporal das aves submetidas à ventilação forçada nos primeiros 10 minutos, com temperaturas em torno de $29^{\circ} \mathrm{C}$ e umidade relativa próxima de $66 \%$. Na pesquisa em questão, a maior redução ocorreu na faixa crítica de temperatura, e nos maiores intervalos de espera, a diferença de temperatura retal alcançou $2^{\circ} \mathrm{C}$. Tal resultado foi superior aos encontrados por Hunter et al. (1997), sendo obtida, em pesquisa no Reino Unido, uma redução de $0,9^{\circ} \mathrm{C}$ em condição de espera climatizada.

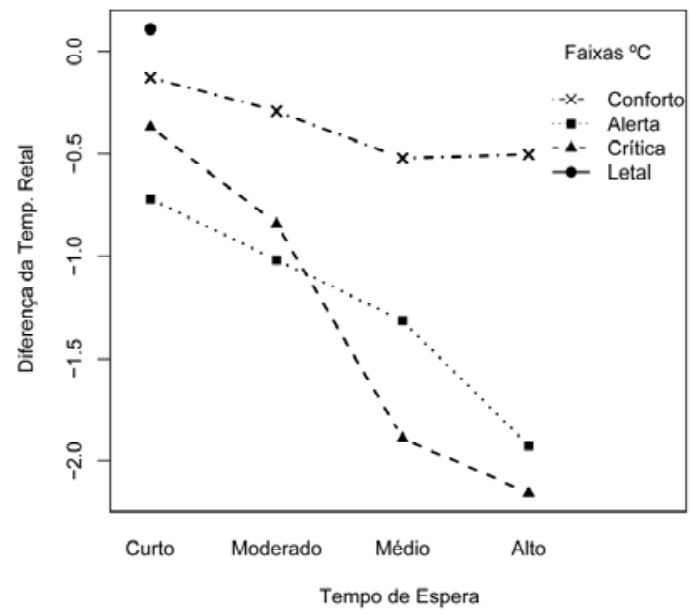

Fig. 2. Valores médios entre a temperatura do galpão de espera e os diferentes intervalos de tempo de espera pré-abate de frangos de corte, sendo intervalos de tempo de espera: curto: abaixo de uma hora; moderado: entre uma e duas horas; médio: entre duas e três horas; alto: acima de três horas, e as faixas de temperatura: conforto: abaixo de $21^{\circ} \mathrm{C}$; alerta: $22-24^{\circ} \mathrm{C}$; crítica: $25-28^{\circ} \mathrm{C}$; letal: acima de $28^{\circ} \mathrm{C}$.

É evidente que o controle térmico do ambiente, por meio da observação da temperatura do ar e da umidade relativa, proporciona maior conforto para os animais dentro dos caminhões, além de reduzir as perdas por mortalidade. No entanto, um importante complemento para esse controle é o tempo de espera, uma vez que, em condição de climatização, a maior permanência dentro do galpão de espera nessas condições possibilita maior troca de calor das aves com o ambiente (Silva e Vieira, 2010).

\section{CONCLUSÃO}

Com relação à interação entre temperatura e umidade relativa interna do galpão de espera, houve eficiência do ambiente climatizado no abatedouro quanto à diminuição do número de aves mortas. A climatização foi mais expressiva na redução do número de aves mortas quando a temperatura do galpão de espera se encontrou entre 21 e $24^{\circ} \mathrm{C}$, principalmente em condições de umidade relativa interna em torno de $70 \%$. Em situação de valores de temperatura entre 22 e $28^{\circ} \mathrm{C}$, a redução da temperatura retal foi mais efetiva quando o tempo de espera foi superior a duas horas. 


\section{AGRADECIMENTOS}

Os autores agradecem à Fundação de Amparo à Pesquisa do Estado de São Paulo (Fapesp) pela concessão da bolsa de estudo.

\section{REFERÊNCIAS}

AKŞIT, M.; YALÇIN, S.; ÖZKAN, S. et al. Effects of temperature during rearing and crating on stress parameters and meat quality or broilers. Poult. Sci., v.85, p.1867-1874, 2006.

BALNAVE, D. Increased utilization of sensible heat loss mechanisms in high temperature, high humidity conditions. World's Poult. Sci. J., v.54, p.69-72, 1998.

BARBOSA FILHO, J.A.D.; VIEIRA, F.M.C.; SILVA, I.J.O. et al. Transporte de frangos: caracterização do microclima da carga durante o inverno. Rev. Bras. Zootec., v.38, p.2442-2446, 2009.

BAYLISS, P.A.; HINTON, M.H. Transportation of broilers with special reference to mortality rates. Appl. Anim. Behav. Sci., v.28, p.93-118, 1990.

BIANCHI, M.; PETRACCI, M.; CAVANI, C. Effects of transport and lairage on mortality, liveweight loss and carcass quality in broiler chickens. Ital. J. Anim. Sci., v.4, p.516-518, 2005.

BRESSAN, M.C.; BERAQUET, N.J. Efeito de fatores pré-abate sobre a qualidade da carne de peito de frango. Ciênc. Agrotec., v.26, p.10491059, 2002.

CLIMA e hidrologia. São Carlos: Embrapa Pecuária Sudeste, 2007. Disponível em: $<$ http://www.cppse.embrapa.br/servicos/dadosmeteorologicos>. Acessado em: 02 de fev. 2007.

CURTIS, S.E. Environmental management in animal agriculture. Ames: The Iowa State University, 1983. 409 p.

DUNN, P.K.; SMYTH, G.K. Double generalized linear models: dglm. R package. Version 1.6.1. 2006. Disponível em: <https://www.rproject.org>. Acessado em: 06/2014.
FURLAN, R.L.; MACARI, M.; SECATO, E.R. et al. Air velocity and exposure time to ventilation affect body surface and rectal temperature of broiler chickens. J. Appl. Poult. Res., v.9, p.1-5, 2000.

GREGORY, N.G. How climatic changes could affect meat quality. Food Res. Int., v.4, p.18661873, 2010.

HUNTER, R.R.; MITCHELL, M.A.; MATHEU, C. Distribution of "dead on arrivals" within the bio-load on commercial broiler transporters: correlation with climatic conditions and ventilation regimen. Br. Poult. Sci., v.38, p.7-S, 1997.

KETTLEWELL, P.J.; HOXEY, R.P.; MITCHELL, M.A. Heat produced by broiler chickens in a commercial transport vehicle. $J$. Agric. Eng. Res., v.75, p.315-326, 2000.

KNIGHT, K. Mathematical statistics. Boca Raton: Chapman e Hall, 2000.

KRANEN, R.W.; VEERKAMP, C.H.; LAMBOOY, E. et al. The effect of thermal preslaughter stress on the susceptibility of broiler chickens differing with respect to growth rate, age at slaughter, blood parameters, and ascites mortality, to hemorrhages in muscles. Poultry Sci., v.77, p.737-744, 1998.

MACARI, M.; FURLAN, R.L. Ambiência aplicada na produção de aves em clima tropical. In: SILVA, I.J.O. (Ed.). Ambiência aplicada na produção de aves em clima tropical. Piracicaba: SBEA, 2001. 200 p.

McCULLAGH, P.; NELDER, J.A. Generalized linear models. London: Chapman and Hall, 1989.

MITCHELL, M.A.; KETTLEWELL, P.J. Road transportation in broiler chickens: induction of physiological stress. World's Poult. Sci. J., v.50, p.57-59, 1994.

MITCHELL, M.A.; KETTLEWELL, P.J.; Physiological stress and welfare of broiler chickens in transit: solutions not problems! Poult. Sci., v.77, p.1803-1814. 1998.

NIJDAM, E.; ARENS, P.; LAMBOOIJ, E. et al. Factors influencing bruises and mortality of broilers during catching, transport, and lairage. Poult. Sci., v.83, p.1610-1615, 2004. 
R DEVELOPMENT core team. $R$ : A language and environment for statistical computing. Vienna: R Foundation for Statistical Computing, 2006. Disponível em: http://www.R-project.org. Acesso em 02 fev. 2007.

RITZ, C.W.; WEBSTER, A.B.; CZARICK, M. Evaluation of hot weather thermal environment and incidence of mortality associated with broiler live haul. J. Appl. Poult. Res., v.14, p.594-602, 2005.

SILVA, I.J.O.; VIEIRA, F.M.C. Ambiência animal e as perdas produtivas no manejo préabate: o caso da avicultura de corte brasileira. Arch. Zootec., v.59, p.113-131, 2010.

SILVA, I.J.O; VIEIRA, F.M.C. Manejo préabate de frangos de corte em dias frios: os cuidados com a ambiência e bem-estar das aves transportadas durante o inverno. Thesis, v.17, p.79-90, 2012.

SILVA, R.G. Introdução à bioclimatologia animal. São Paulo: Nobel, 2000. 288 p.

SIMÕES, G.S.; OBA, A.; MATSUO, T. et al. Vehicle thermal microclimate evaluation during Brazilian summer broiler transport and the occurrence of PSE (pale, soft, exudative) meat. Braz. Arch. Biol. Technol., v.52, p.195-204, 2009.
VIEIRA, A.M.C. Modelagem simultânea de média e dispersão e aplicações na pesquisa agronômica. 2008. 117f. Tese (Doutorado em Agronomia) - Escola Superior de Agricultura Luiz de Queiroz, Piracicaba, SP.

VIEIRA, F.M.C.; SILVA, I.J.O.; BARBOSA FILHO, J.A.D. et al. Productive losses on broiler preslaughter operations: effects of the distance from farms to abattoirs and of lairage time in a climatized holding area. Rev. Bras. Zootec., v.39, p.2471-2476, 2010 .

VIEIRA, F.M.C.; SILVA, I.J.O.; BARBOSA FILHO, J.A.D. et al. Thermal stress related with mortality rates on broiler's preslaughter operations: a lairage time effect study. Ciênc. Rural, v.41, p.1639-1644, 2011 a.

VIEIRA, F.M.C.; SILVA, I.J.O.; BARBOSA FILHO, J.A.D. et al. Preslaughter mortality of broilers in relation to lairage and season in a subtropical climate. Poult. Sci., v.90, p. 21272133, 2011b.

WEBSTER, A.J.F.; TUDDENHAM, A.; SAVILLE, C.A. et al. Thermal stress on chickens in transit. Br. Poult. Sci., v.34, p.267$277,1993$. 